\title{
Evaluating Equivocal Left Main Stenosis with Fractional Flow Reserve - Not all Daughter Vessels are Created Equally
}

\author{
Ahmed Almomani, ${ }^{1}$ Abdul-Rahman Abdel-Karim, ${ }^{2}$ Barry F Uretsky ${ }^{3,4}$ \\ 1. University of Texas Health San Antonio, San Antonio, TX, USA; 2. Methodist Le Bonheur Healthcare System, Memphis, TN, USA \\ 3. University of Arkansas for Medical Sciences, Little Rock, AR, USA; 4. Central Arkansas Veterans Healthcare System, Little Rock, AR, USA
}

DOl: https://doi.org/10.17925/HI.2019.13.2.38

$\mathrm{F}$ ractional flow reserve (FFR) evaluation of intermediate left main coronary artery (LMCA) stenosis has been validated in clinical decision-making. However, the size of the daughter vessel, in which the FFR transducer is placed and the amount of myocardium it subtends, has received less attention. We present a case that demonstrates the importance of transducer location, size of the daughter vessel and the amount of subtended myocardium in evaluation of LMCA stenosis, pointing out potential pitfalls.

\section{Keywords}

Left main disease, fractional flow reserve, coronary revascularisation, intravascular ultrasound, angiography

Disclosure: Ahmed Almomani, Abdul-Rahman Abdel-Karim and Barry Uretsky have nothing to disclose in relation to this article.

Review Process: Double-blind peer review.

Compliance with Ethics: Written informed consent was not obtained from the patient case included in this report; however, no identifying information or images have been used.

Authorship: The named authors meet the International Committee of Medical Journal Editors (ICMJE) criteria for authorship of this manuscript, take responsibility for the integrity of the work as a whole, and have given final approval for the version to be published.

Received: 3 April 2019

Accepted: 5 November 2019

Citation: Heart International. 2019;13(2):38-40

Corresponding Author: Barry F Uretsky,

Central Arkansas Veterans Healthcare System,

4300 West Seventh Street, Little Rock, AR 72205

USA. E: buretsky@gmail.com

Support: No funding was received in the publication of this article.
Fractional flow reserve (FFR) has been a useful tool in guiding decision-making in intermediate left main coronary artery (LMCA) stenosis, particularly in the absence of downstream disease. Multiple caveats have been raised about the use of FFR with significant downstream disease and the interpretation of the significance of the LMCA stenosis. ${ }^{2}$ On the other hand, very little emphasis has been placed on the importance of the FFR transducer location and the amount of myocardium subtended, presumably because, in most cases, the left anterior descending (LAD) and left circumflex (CX), both supply a large amount of myocardium that would easily clarify whether blood flow is limited from a LMCA stenosis. However, this may not always be true as illustrated by this case.

\section{Case report}

A 69-year-old man was referred for angiography for Canadian Cardiovascular Society (CCS) class III angina. He previously had stents implanted in his right coronary artery, LAD and first obtuse marginal branch. A coronary angiogram showed intermediate LMCA stenosis (Figure 1). Due to severe radial artery spasm and vessel tortuosity, catheter manipulation was challenging. As such, it was elected to perform FFR of the LMCA through the diagnostic catheter. The pressure wire could only be advanced into the ramus intermedius (RI) and pressure transducer for few millimetres distal to the lesion, and intravenous adenosine (140 $\mathrm{mcg} / \mathrm{kg} / \mathrm{min})$ was administered to achieve haemodynamic response; FFR was 0.87 (Figure 2). On the basis of the unchanged coronary anatomy, compared to a previous angiogram 3 years earlier, except for mild angiographic progression of the LMCA stenosis and the non-ischaemic FFR value, it was elected to treat the patient medically.

One month later, the patient reported improved but persistent CCS II angina. On the basis of the symptoms and angiographic severity of the left main lesion, the patient was brought back for FFR and intravascular ultrasound of the LMCA lesion through the femoral approach to overcome the technical difficulties faced in the previous procedure. FFR of the LMCA lesion was performed in all three daughter vessels ( $L A D, C X$ and RI). Again, FFR was non-ischaemic (0.86) in the RI consistent with the previous assessment with the pressure wire greater than $10 \mathrm{~mm}$ distal to the lesion. However, FFR was in the ischaemic range for both the LAD and the Cx (Table 1, Figure 3). Intravascular ultrasound of LMCA showed a minimal luminal area of $5.6 \mathrm{~mm}^{2}$ (Figure 4). The patient was referred for coronary artery bypass surgery.

\section{Discussion}

FFR-guided percutaneous coronary intervention in non-left main lesions has shown better long-term clinical outcomes compared to angiography alone in stable ischaemic heart disease. ${ }^{3,4}$ Furthermore, numerous studies support the use of FFR to assess intermediate LMCA coronary stenosis. ${ }^{1.5-7}$ However, as with any diagnostic test, the technique and limitations of its applications should be understood.

The reliability of FFR for simple LMCA lesions is rarely an issue, but interpreting the LMCA FFR in the presence of significant downstream branch lesions, such as an LAD stenosis, is a 
Figure 1: Intermediate left main coronary artery disease with estimated stenosis of $60 \%$

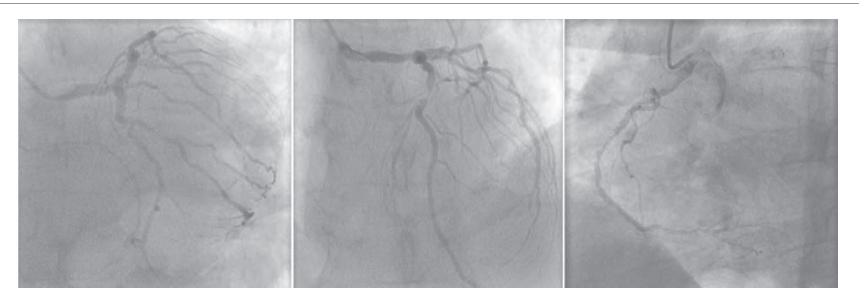

Figure 2: Fractional flow reserve in ramus intermedius on initial study
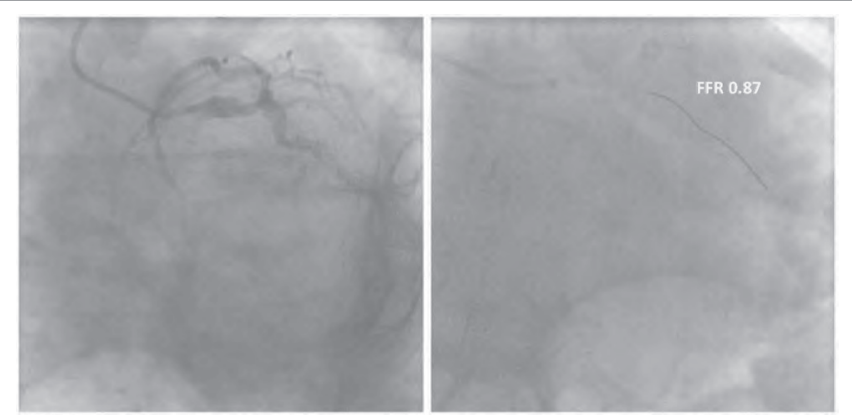

FFR was 0.87 in the ramus intermedius. $F F R=$ fractional flow reserve.

Table 1: Fractional flow reserve across the left main stenosis in all three daughter vessels

\begin{tabular}{|l|l|l|l|}
\hline Artery & Baseline Pd/Pa & FFR in the distal vessel & Pressure drift \\
\hline $\begin{array}{l}\text { Left anterior } \\
\text { descending }\end{array}$ & 0.82 & 0.62 & None \\
\hline $\begin{array}{l}\text { Ramus } \\
\text { intermedius }\end{array}$ & 0.93 & 0.86 & None \\
\hline Left circumflex & 0.92 & 0.75 & None \\
\hline
\end{tabular}

$F F R=$ fractional flow reserve; $P d / P a=$ pressure distal to lesion/aortic pressure.

recognised challenge. LMCA and the downstream lesions act as serial lesions, and the true flow across the LMCA is potentially reduced by a severe downstream stenosis, resulting in false elevation of the LMCA FFR when measured in the unobstructed vessel. ${ }^{2.8}$ Less emphasis has been placed on the importance of the location of the FFR transducer in the absence of downstream stenosis and its relation to the amount of myocardium supplied by the daughter branch. ${ }^{9}$ For an accurate FFR, maximal hyperaemia must be achieved across the LMCA stenosis. Flow through the LMCA is the sum of the flow in all daughter branches, the magnitude of flow being proportional to the size of each artery's viable myocardial bed.

This case points out that the size of the daughter vessel and the amount of myocardium it subtends is a factor in determining the measured FFR. In this particular case, the RI was small with a small subtended myocardium, and despite the LMCA lesion, the blood flow required during maximal hyperaemia was adequate. In small coronary arteries pressure recovery occurs within $1-2 \mathrm{~mm}$ distal to stenosis, ${ }^{10}$ and the fact that the transducer could only be advanced a few millimetres distal to the lesion should not have affected the FFR measurement. Additionally, the fact that the FFR value on the second study was
Figure 3: Fractional flow reserve of daughter vessels

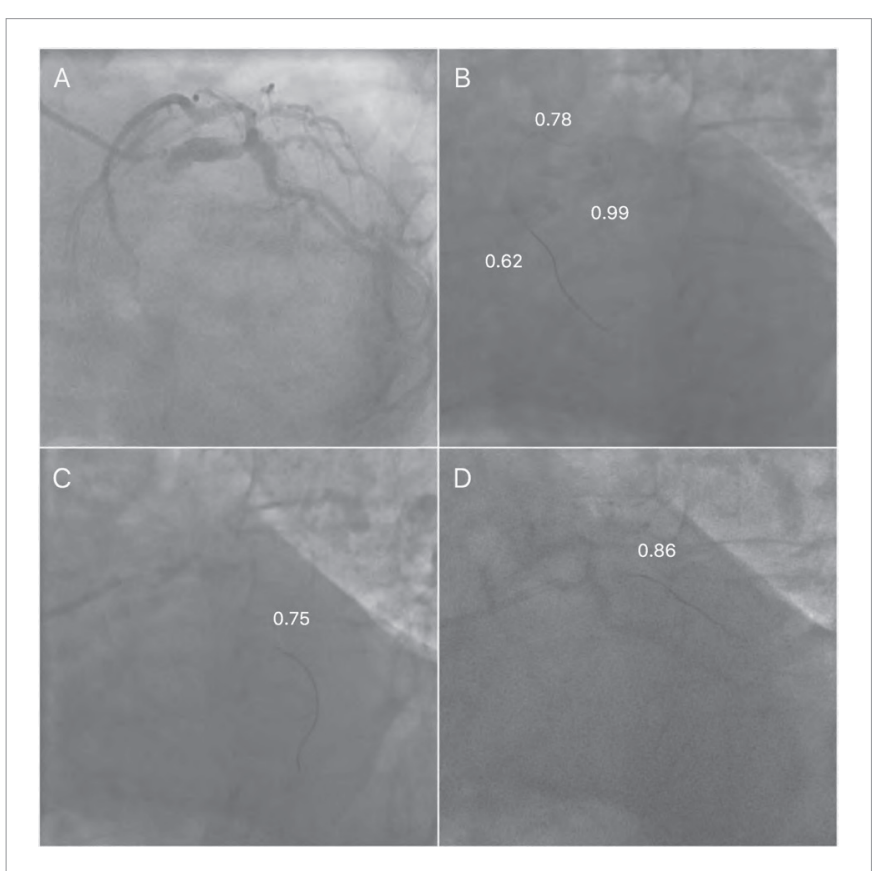

Fractional flow reserve of daughter vessels 3 months later showing ischaemic fractional flow reserve in left anterior descending (3B) and in left circumflex (3C), but non-ischaemic fractional flow reserve in ramus intermedius (3D).

Figure 4: Intravascular ultrasound of the left main coronary artery demonstrating a minimal lumen area of $5.6 \mathrm{~mm}^{2}$

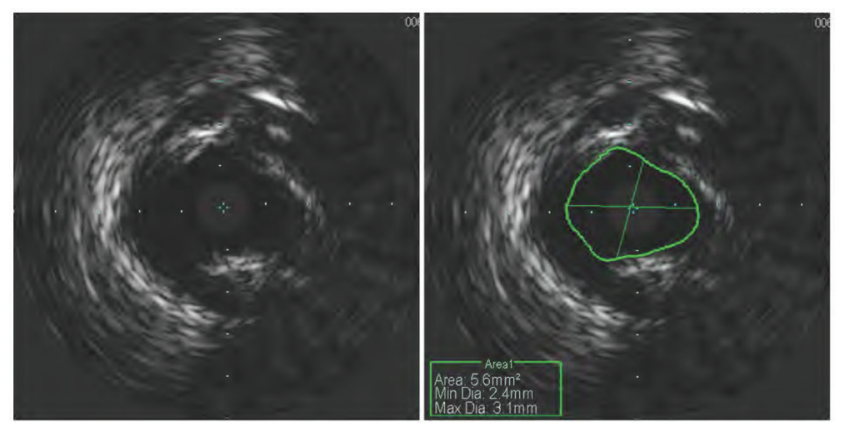

essentially the same as the first is in keeping with the accuracy of the first measurement. On the other hand, both the $C x$ and $L A D$ were larger vessels with larger subtended myocardium and both showed ischaemic FFR. Thus, it is important to keep in mind that the pressure transducer needs to be in a larger daughter vessel of the LMCA when measuring FFR in order to be used appropriately for clinical decision-making.

\section{Conclusion}

FFR is well accepted as a valuable tool in clinical decision-making of intermediate left main stenosis. This case illustrates an important consideration in its use. Specifically, if the pressure transducer is in a small daughter vessel, typically a small RI or CX, the FFR value may be in the non-ischaemic range due to the limited area of myocardium subtended by the vessel when, in fact, FFR measured in a larger vessel may show ischaemia. The case emphasises the need to measure FFR in all the daughter vessels, especially those with the largest myocardial territory. • 
1. Hamilos $\mathrm{M}$, Muller $\mathrm{O}$, Cuisset T, et al. Long-term clinical outcome after fractional flow reserve-guided treatment in patients with angiog artery stenosis. Circulation. 2009;120:1505-12.

2 Daniels DV van't Veer M, Pijls NH, et al. The impact of downstream coronary stenoses on fractional flow reserve assessment of intermediate left main disease. JACC CardiovasC Interv. 2012;5:1021-5.

3. De Bruyne B, Pijls NH, Kalesan B, et al. Fractional flow reserve-guided $\mathrm{PCl}$ versus medical therapy in stable coronary disease. N Engl J Med. 2012;367:991-1001.

4. Pijls NH, Fearon WF, Tonino PA, et al. Fractional flow reserve versus angiography for guiding percutaneous coronary intervention in patients with multivessel coronary artery disease: 2-year follow-up of the FAME (fractional flow reserve versus angiography for multivessel evaluation) study. J Am Coll Cardiol. 2010;56:177-84.

5. Bech GJ, Droste H, Pijls NH, et al. Value of fractional flow reserve in making decisions about bypass surgery for equivocal left mar 2001; 86:547-52

6. Jasti V, Ivan $E$, Yalamanchili V, et al. Correlations between fractional flow reserve and intravascular ultrasound in patients with an ambiguous left main coronary artery stenosis Circulation. 2004;110:2831-6.

7. Lindstaedt $M$, Yazar A, Germing A, et al. Clinical outcome in patients with intermediate or equivocal left main coronary artery disease after deferral of surgical revascularization on the basis of fractional flow reserve measurements. Am Heart s. 2006;152:156.e1-9.

8. Fearon WF, Yong AS, Lenders $G$, et al. The impact of downstream coronary stenosis on fractional flow reserve assessment of intermediate left main coronary artery disease: human validation. JACC Cardiovasc Interv. 2015;8:398-403

9. Echavarría-Pinto M, van de Hoef TP, Nijjer S, et al. Influence of the amount of myocardium subtended to a coronary stenosis on the index of microcirculatory resistance. Implications for the invasive assessment of microcirculatory function in ischaemic heart disease. Eurolntervention. 2017;13:944-52.

10. Ofili EO, Labovitz AJ, Kern MJ. Coronary flow velocity dynamics in normal and diseased arteries. Am J Cardiol. 1993:71:3-9D. 